\title{
Fast and Ultrasensitive Electrometer Operating at the Single-Photon Level
}

\author{
B. L. Brock * Juliang Li $\left.\right|^{\dagger}$ S. Kanhirathingal, B. Thyagarajan, M. P. Blencowe, and A. J. Rimberg ${ }^{\ddagger}$ \\ Department of Physics and Astronomy, Dartmouth College, Hanover, New Hampshire 03755, USA
}

(Dated: October 29, 2021)

\begin{abstract}
We demonstrate fast and ultrasensitive charge detection with a cavity-embedded Cooper pair transistor (cCPT) via dispersive readout of its Josephson inductance. We report a minimum charge sensitivity of $14 \mu e / \sqrt{\mathrm{Hz}}$ with a detection bandwidth on the order of $1 \mathrm{MHz}$ using 16 attowatts of power, corresponding to the single-photon level of the cavity. In addition, our measured sensitivities are within a factor of 5 of the quantum limit for this device. The single-photon-level sensitivity of the cCPT is comparable to that of the rf-SET, which typically operates using picowatts of power corresponding to hundreds of thousands of photons in its tank circuit. Our results support the feasibility of using the cCPT to mediate an optomechanical interaction that reaches the singlephoton strong coupling regime.
\end{abstract}

Fast and ultrasensitive electrometers have been instrumental to the advancement of basic science. They have been used to detect in real time the tunneling of electrons in a quantum dot 1], determine the tunneling rates of quasiparticles in superconducting devices 2, and search for signatures of Majorana zero modes in nanowires [3]. In addition, the rapid detection of single electrons is crucial for the readout of quantum-dot-based qubits [4, for which operating at lower photon numbers reduces measurement backaction [5. In this same vein, ultrasensitive electrometers are at the heart of many schemes for sensing the displacement of charged mechanical resonators [6] 8], as well as for coherently coupling mechanical resonators to microwave cavities 9 -11]. To observe and take advantage of quantum effects in such hybrid systems it is often essential that their coupling be strong at the singlephoton level, a regime that has been achieved for quantum dots [12, 13] but not yet for mechanical resonators despite significant effort 14 17. Reaching the singlephoton strong optomechanical coupling regime, where a single cavity photon causes sufficient radiation pressure to displace the mechanical resonator by more than its zero-point uncertainty, would enable the generation of nonclassical states of both light and motion [18, 19, as well as provide a rich platform for studying the quantumto-classical transition and other fundamental physics 20 .

Electrometers based on the single electron transistor (SET) are among the fastest and most sensitive reported in the literature to date. Radio-frequency single electron transistors (rf-SETs) are the best known of these devices, having achieved sensitivities below $1 \mu e / \sqrt{\mathrm{Hz}} 21$ and bandwidths greater than $100 \mathrm{MHz}$ 22. The rf-SET encodes the charge gating the SET in the power dissipated by the SET, which is embedded in a tank circuit to enable

\footnotetext{
* Benjamin.L.Brock.GR@dartmouth.edu

Present address: Department of Applied Physics, Yale University, New Haven, Connecticut 06520, USA

$\dagger$ Present address: High Energy Physics Divison, Argonne National Laboratory, 9700 South Cass Avenue, Argonne, IL 60439, USA

‡ Alexander.J.Rimberg@dartmouth.edu
}

$\mathrm{RF}$ readout of this dissipation. This dissipative detection typically requires picowatts of power, corresponding to hundreds of thousands of photons in the tank circuit, rendering the rf-SET unsuitable for some of the aforementioned applications and making it impossible to integrate the rf-SET with modern near-quantum-limited amplifiers 23 25 (which typically saturate well below the picowatt scale). Dispersive electrometers based on the SET have also been developed, which encode the gate charge in the resonant frequency of a tank circuit. Such electrometers have been operated using femtowatts of power [2, 26], corresponding to tens or hundreds of photons, and have achieved sensitivities as low as $30 \mu e / \sqrt{\mathrm{Hz}}$ [27]. More recently, dispersive gate-based sensors have been developed 28. that have surpassed the performance of SET-based electrometers. These devices have achieved sensitivities as low as $0.25 \mu e / \sqrt{\mathrm{Hz}}$ with bandwidths approaching 1 $\mathrm{MHz}$ using 100 attowatts of power, corresponding to hundreds of photons 29 .

In this letter we demonstrate ultrasensitive dispersive charge detection with a cavity-embedded Cooper pair transistor (cCPT) 30, 31. Using 16 attowatts of power, corresponding to the single-photon level of the cavity, we measure a minimum charge sensitivity of $14 \mu e / \sqrt{\mathrm{Hz}}$. We find that the cCPT operates within a factor of 5 of its theoretical quantum-limited sensitivity, this discrepancy being due to frequency noise, amplifier noise, and the nonlinearity of the device. Another limitation of the present device is quasiparticle poisoning [32, which prevents us from studying the cCPT at its theoreticallyoptimal operating point. Based on these results we expect an optimized sample could achieve a sensitivity as low as $0.4 \mu e / \sqrt{\mathrm{Hz}}$, rivaling that of the best gate-based sensor [29]. Due to its ability to operate at the singlephoton-level, the cCPT has been proposed as a platform for reaching the single-photon strong coupling regime of optomechanics 9. Our results support the feasibility of this proposal and represent an important step toward its realization.

Here we study the same device characterized experimentally in Ref. [30. The most important parameters of this realization of the cCPT are shown in Table [. For more information on this device, including sample 


\begin{tabular}{|c|c|}
\hline Josephson energy & $E_{J} / h=14.8 \mathrm{GHz}$ \\
\hline Charging energy & $E_{C} / h=54.1 \mathrm{GHz}$ \\
\hline Gate capacitance & $C_{g}=6.3 \mathrm{aF}$ \\
\hline Coupling capacitance & $C_{c}=7.1 \mathrm{fF}$ \\
\hline Bare cavity frequency & $\omega_{\lambda / 4} / 2 \pi \approx 5.76 \mathrm{GHz}$ \\
\hline Cavity linewidth & $\kappa_{\mathrm{tot}} / 2 \pi \approx 1.4 \mathrm{MHz}$ \\
\hline Cavity length & $\ell=5135 \mu \mathrm{m}$ \\
\hline Characteristic impedance & $Z_{0}=50 \Omega$ \\
\hline
\end{tabular}

TABLE I. Parameters of the cCPT [30].

images, fabrication methods, and characterization techniques, see Ref. [30.

The cCPT, depicted schematically in Fig. 11(a), has two components: a quarter-wavelength $(\lambda / 4)$ coplanar waveguide cavity and a Cooper pair transistor (CPT). The CPT consists of two Josephson junctions (JJs) with an island between them that can be gated via the capacitance $C_{g}$. The CPT is connected between the voltage antinode of the cavity and the ground plane, such that the two form a SQUID loop. Embedded in this way, the CPT behaves as a nonlinear Josephson inductance $L_{J}$ in parallel with the cavity that can be tuned by both the number of electrons $n_{g}$ gating the island and the flux $\Phi_{\text {ext }}$ threading the SQUID loop. The gate charge $n_{g}$ is thus encoded in the resonant frequency $\omega_{0}$ of the cavity, which can then be detected via microwave reflectometry. The theoretical charge sensitivity of the cCPT in this mode of operation is derived from first principles in Ref. 31. This device can be operated at much lower powers than comparable SET-based dispersive electrometers [2, 26, 27, for two key reasons. First, we use a distributed superconducting microwave cavity rather than a lumped-element LC circuit, yielding much lower dissipation. Second, we can tune the CPT band structure via the external flux $\Phi_{\text {ext }}$, which provides us greater flexibility in biasing the device to an optimally-sensitive point.

To measure the charge sensitivity of the cCPT we drive the cavity with a resonant carrier signal while modulating the gate about a dc bias point $n_{g}$ such that $n_{g}(t)=n_{g}+\sqrt{2}\left(q_{\mathrm{rms}} / e\right) \cos \left(\omega_{g} t\right)$, which in turn modulates the resonant frequency according to $\omega_{0}(t)=$ $\omega_{0}+\sqrt{2}\left(\partial \omega_{0} / \partial n_{g}\right)\left(q_{\mathrm{rms}} / e\right) \cos \left(\omega_{g} t\right)$. As a result, the reflected carrier signal is phase-modulated leading to output power $P_{\text {out }}$ proportional to $q_{\mathrm{rms}}^{2}$ at the sideband frequencies $\omega_{0} \pm \omega_{g}$. Thus, given the rms charge modulation amplitude $q_{\mathrm{rms}}$, we can use a spectrum analyzer with resolution bandwidth $B$ to measure the sidebands and thereby extract the charge sensitivity $\delta q$ from

$$
\delta q=\frac{q_{\mathrm{rms}}}{\sqrt{2 B} \times 10^{\mathrm{SNR} / 20}},
$$

where SNR is the single-sideband signal to noise ratio expressed in decibels [21, 34]. Here we consider the total power at the two sidebands to be the signal of interest, since it is possible to combine them via homodyne mix-
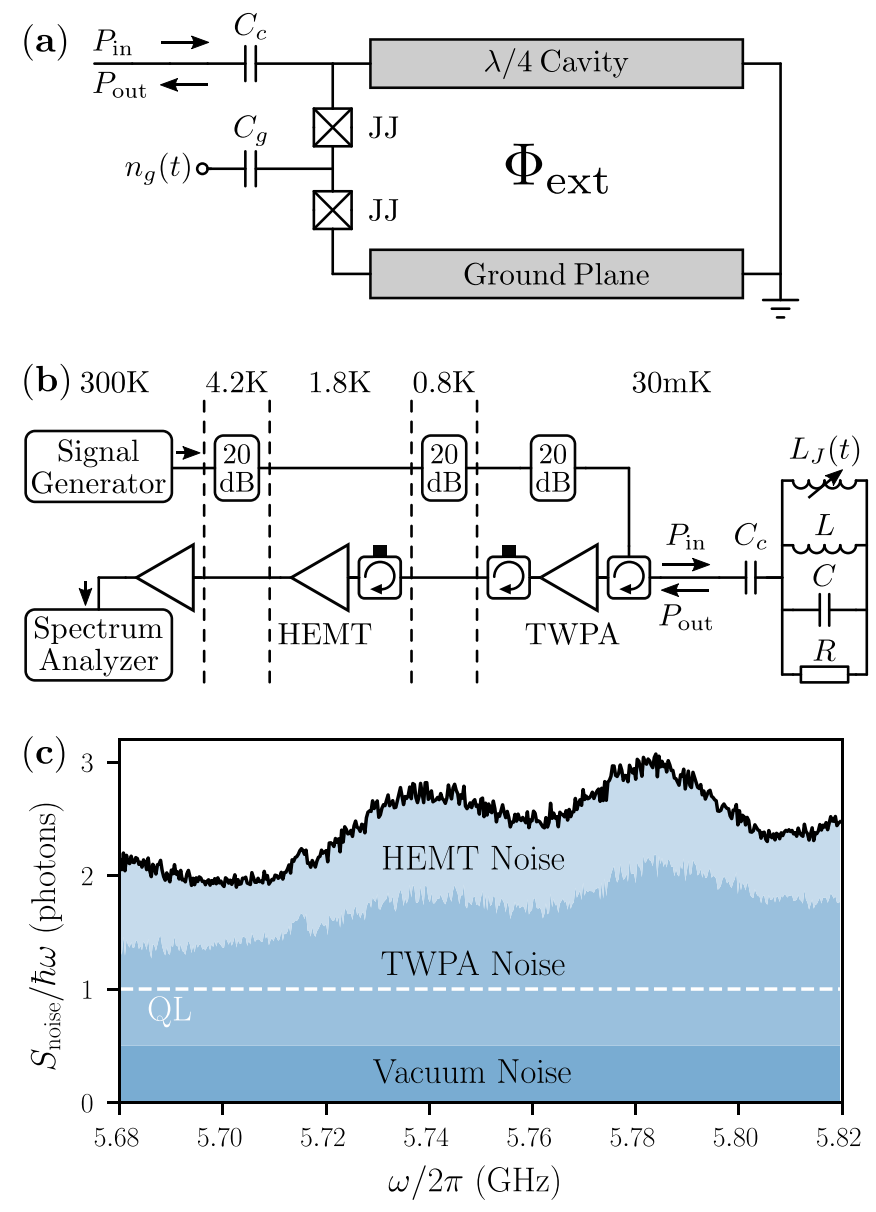

FIG. 1. (a) Schematic of the cCPT. (b) Schematic of the measurement circuitry. The cavity behaves as a parallel RLC circuit when driven near its fundamental frequency 33], and the CPT behaves as an inductance $L_{J}$ in parallel with the cavity. (c) System noise referred to the sample plane (solid black line). Shaded areas show the contribution of each noise source. The dashed white line is the quantum limit.

ing, leading to the factor of $1 / \sqrt{2}$ above. Theoretically, the output sideband power $P_{\text {out }}\left(\omega_{0} \pm \omega_{g}\right)$ can be expressed

$$
P_{\text {out }}\left(\omega_{0} \pm \omega_{g}\right)=\frac{2 \kappa_{\text {ext }}^{2}}{\kappa_{\text {tot }}^{2}\left(\omega_{g}^{2}+\kappa_{\text {tot }}^{2} / 4\right)}\left|\frac{q_{\mathrm{rms}}}{e} \frac{\partial \omega_{0}}{\partial n_{g}}\right|^{2} P_{\text {in }},
$$

where $\kappa_{\text {ext }}$ and $\kappa_{\text {tot }}$ are the external and total damping rates of the cavity, respectively, and $P_{\text {in }}$ is the input carrier power at the plane of the sample [30]. The theoretical charge sensitivity can therefore be expressed as 31.

$$
\delta q=\frac{\kappa_{\text {tot }}}{2 \kappa_{\text {ext }}} \sqrt{\frac{S_{\text {noise }}}{P_{\text {in }}}\left(\omega_{g}^{2}+\frac{\kappa_{\text {tot }}^{2}}{4}\right)}\left|\frac{\partial \omega_{0}}{\partial n_{g}}\right|^{-1} e .
$$

To evaluate this expression we use the sample-referred $S_{\text {noise }}$ and $P_{\text {in }}$ (discussed below), as well as the values of $\kappa_{\text {ext }}, \kappa_{\text {tot }}$, and $\omega_{0}\left(n_{g}, \Phi_{\text {ext }}\right)$ determined from a detailed characterization of the device [30. The damping rates are approximately $\kappa_{\text {ext }} / 2 \pi \approx 1.2 \mathrm{MHz}$ and $\kappa_{\text {tot }} / 2 \pi \approx$ 
$1.4 \mathrm{MHz}$, though these depend on $\omega_{0}\left(n_{g}, \Phi_{\text {ext }}\right)$ and can vary by $10 \%-20 \%$. The corresponding quantum-limited sensitivity of the device is obtained by evaluating Eq. (3) at the quantum limit of system noise for our measurement scheme, $S_{\text {noise }}^{\mathrm{QL}}=\hbar \omega$, as discussed below.

Importantly, both Eqs. (1) and (3) are only valid when $q_{\mathrm{rms}} / e \ll \omega_{g} /\left(\partial \omega_{0} / \partial n_{g}\right)$, which ensures that the amplitude of the resulting frequency modulation is small compared to $\omega_{g}$ and that $P_{\text {out }}\left(\omega_{0} \pm \omega_{g}\right) \propto q_{\text {rms }}^{2}$. In all of our measurements we use sufficiently small $q_{\mathrm{rms}}$ to satisfy this constraint. Furthermore, Eq. (3) is most accurate in the linear response regime for which $n \ll \kappa_{\text {tot }} /|K|$, where $n=4 \kappa_{\text {ext }} P_{\text {in }} / \hbar \omega_{0} \kappa_{\text {tot }}^{2}$ is the average number of intracavity photons and $K$ is the Kerr nonlinearity of the cCPT [30]. Experimentally, we find that for $n \ll \kappa_{\text {tot }} /|K|$ the output sideband power grows linearly with $P_{\text {in }}$ as expected from Eq. (2), but as $n$ approaches $\kappa_{\text {tot }} /|K|$ this trend becomes sub-linear. Near this threshold, $P_{\text {out }}\left(\omega_{0} \pm \omega_{g}\right)$ begins to decrease with increasing $P_{\text {in }}$. For the present device this threshold corresponds to the single-photon-level [30, so we perform all of our measurements with $n \lesssim 1$. In this sense the single-photon-level operation of the cCPT can be viewed as both an enabling feature (for the reasons described earlier) and a constraint, but this constraint could be avoided in future devices by changing $E_{J}, E_{C}$, and $\kappa_{\text {tot }}$.

The detection bandwidth of the present device, which determines the maximum rate at which the cavity can respond to changes in $n_{g}$, is set by $\kappa_{\text {tot }}$ and is on the order of $1 \mathrm{MHz}$. The bandwidth can be improved by increasing the coupling capacitance $C_{c}$, thereby increasing $\kappa_{\text {ext }}$, but this also affects the single-photon-level charge sensitivity. Setting $n=1$ and assuming negligible internal loss such that $\kappa_{\text {tot }} \approx \kappa_{\text {ext }}$, Eq. (3) predicts $\delta q \propto \sqrt{\kappa_{\text {tot }}}$ for $\omega_{g} \ll \kappa_{\text {tot }}$. However, if we restrict ourselves to the linear-response regime rather than the single-photon level we can operate with $n \sim \kappa_{\text {tot }} /|K|$, in which case $\delta q$ is independent of $\kappa_{\text {tot }}$. Lastly, it is worth noting that if one increases $\kappa_{\text {ext }}$, one also increases the charge noise coupling to the cCPT via the input-output transmission line [31, which is negligible in the present device.

The cCPT is housed in a dilution refrigerator with a base temperature of $T \lesssim 30 \mathrm{mK}$ and measured using the circuitry depicted schematically in Fig. 1(b), which is nearly identical to that used in Ref. [30]. The one difference here is that we use a near quantum-limited TWPA [24] as a first-stage amplifier. We use the techniques described in Ref. 30. to refer all input and output powers, as well as the system noise $S_{\text {noise }}(\omega)$, to the plane of the sample. The measured system noise, shown in Fig. 1(c), is due to the half-photon of vacuum noise $S_{\text {vac }}=\hbar \omega / 2$ in the input/output transmission line 35. and the added noise of our amplifier chain $S_{\text {amp }}$, such that $S_{\text {noise }}=S_{\text {vac }}+S_{\mathrm{amp}}$. For all of the charge sensitivity measurements we report, the noise floor near the sideband frequencies is dominated by this system noise, which is why we use the same notation for these two quantities. At sufficiently low gate modulation frequen-
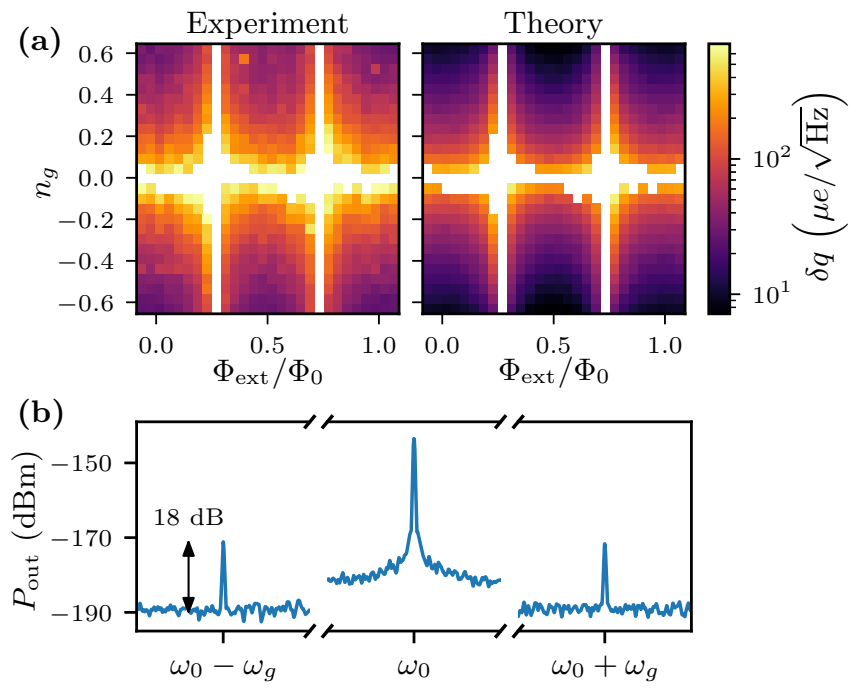

FIG. 2. (a) Measured and theoretical charge sensitivities, obtained using Eqs. (1) and (3) respectively, as a function of gate and flux. Data is omitted where the sidebands could not be resolved from the noise floor. (b) Sample-referred spectrum analyzer trace of the optimal charge sensitivity measurement, corresponding to $\delta q=14 \mu e / \sqrt{\mathrm{Hz}}$. The carrier frequency is $\omega_{0} / 2 \pi=5.806 \mathrm{GHz}$, the gate modulation frequency is $\omega_{g} / 2 \pi=350 \mathrm{kHz}$, the span of each segment is 1 $\mathrm{kHz}$, and the resolution bandwidth is $B=10 \mathrm{~Hz}$. The noise floor near the carrier is due to $1 / f$ charge noise [30, 36].

cies, however, the noise floor is dominated by $1 / f$ charge noise [36]. This regime occurs below about $1 \mathrm{kHz}$ in our case [30. We determine the noise added by the TWPA and HEMT independently by measuring the gain of the amplifier chain and total system noise twice: once with the TWPA pump on and once with it off. Over the operating range of the cCPT (between $5.68 \mathrm{GHz}$ and 5.82 $\mathrm{GHz})$, the TWPA contributes 1.2 photons of noise $(50 \%$ of total) while the HEMT contributes 0.7 photons $(30 \%$ of total) on average. The room temperature amplifier contributes negligibly to the sample-referred system noise $S_{\text {noise }}$. The quantum limit of noise in this system is one photon, such that $S_{\text {noise }}^{\mathrm{QL}}=\hbar \omega$, since phase-insensitive amplifiers must add at least a half-photon of noise 37 . Thus, our average system noise is only a factor of 2.4 greater than the quantum limit for this measurement scheme, such that the theoretical sensitivity (Eq. 3) is only a factor of $\sqrt{2.4}$ greater than the quantum-limited sensitivity.

In order to compare the cCPT's charge sensitivity with its theoretical performance, given by Eq. (3), we first measure $\delta q$ as a function of both the gate charge $n_{g}$ and external flux $\Phi_{\text {ext }}$. Although we can access a full period of $\Phi_{\text {ext }}$ (from 0 to the magnetic flux quantum $\Phi_{0}$ ), we can only access the gate range $-0.65<n_{g}<0.65$ due to quasiparticle poisoning [30]. We perform this measurement using an input power $P_{\text {in }}=-141 \mathrm{dBm}$ $\approx 8 \mathrm{aW}$ and gate modulation amplitude $q_{\mathrm{rms}}=10^{-3} e$. Ideally we would set $\omega_{g}$ to be significantly less than 


\begin{tabular}{|c|c|c|c|}
\hline Electrometer & $\delta q(\mu e / \sqrt{\mathrm{Hz}})$ & $P_{\text {in }}(\mathrm{aW})$ & $n$ photons \\
\hline $\mathrm{cCPT}^{*}$ & 14 & 16 & 1 \\
\hline Best gate sensor [29]* & 0.25 & 100 & 190 \\
\hline Best rf-SET 21] & 0.9 & $6 \times 10^{6}$ & $2 \times 10^{5}$ \\
\hline Andresen et al. 39] & 2.3 & $3 \times 10^{8}$ & $2 \times 10^{6}$ \\
\hline L-SET[27]* & 30 & $1 \times 10^{4}$ & 70 \\
\hline Naaman et al. [2]* & 52 & $2 \times 10^{3}$ & 150 \\
\hline Bell et al. [40]* & 70 & $3 \times 10^{7}$ & $2 \times 10^{5}$ \\
\hline rf-QPC [1] & 200 & $1 \times 10^{9}$ & $7 \times 10^{7}$ \\
\hline
\end{tabular}

TABLE II. Comparison of the cCPT with a representative set of fast and ultrasensitive electrometers. Asterisks indicate dispersive electrometers.

$\kappa_{\text {tot }} / 2 \approx 2 \pi \times 700 \mathrm{kHz}$ to minimize Eq. (3), but in our experiments we observe cross-talk between our gate and flux lines at frequencies below about $650 \mathrm{kHz}$. We therefore use $\omega_{g} / 2 \pi=800 \mathrm{kHz}$, such that the gate modulation does not also induce a flux modulation. To measure the reflected power and noise floor at $\omega_{0} \pm \omega_{g}$ we use a resolution bandwidth $B=1 \mathrm{~Hz}$.

The results of this measurement are shown in Fig. 2(a). We find that the variation of $\delta q$ with $n_{g}$ and $\Phi_{\text {ext }}$ is in good agreement with theory, but our measured sensitivities are about 3 times worse than theory. We attribute this discrepancy to two factors. First and foremost, the resonant frequency fluctuates due to $1 / f$ charge and flux noise [30, 38] over the course of each measurement, which means our carrier is not always on resonance. On average, this reduces the output sideband power yielding worse charge sensitivity than expected. Second, we used a sufficiently high input power that $P_{\text {out }}\left(\omega_{0} \pm \omega_{g}\right)$ scales sublinearly with $P_{\text {in }}$ due to the Kerr nonlinearity. Although this improves the sensitivity overall and was necessary to resolve the sidebands over a large area of the gate/flux parameter space, it causes the measured sensitivity to diverge from theory since the latter assumes $P_{\text {in }} \propto P_{\text {out }}\left(\omega_{0} \pm \omega_{g}\right)$. Finally, since $S_{\text {noise }} / S_{\text {noise }}^{\mathrm{QL}} \approx 2.4$, the factor of 3 discrepancy between theory and experiment means our measured sensitivities are within a factor of 5 of the quantum limit. In this measurement we find a minimum charge sensitivity of $24 \mu e / \sqrt{\mathrm{Hz}}$ at $\left(n_{g}, \Phi_{\text {ext }}\right)=(0.63,0.0)$, whereas our predicted theoretical and quantum-limited sensitivities at this point are $9 \mu e / \sqrt{\mathrm{Hz}}$ and $6 \mu e / \sqrt{\mathrm{Hz}}$, respectively.

In order to optimize $\delta q$ we narrow our search to the gate range $0.6 \leq\left|n_{g}\right| \leq 0.65$ and the flux points $\Phi_{\text {ext }}=$ $0, \Phi_{0} / 2$. At these flux points the resonant frequency of the cCPT is insensitive to flux, so we can reduce our gate modulation frequency to $\omega_{g} / 2 \pi=350 \mathrm{kHz}$ without the gate/flux cross-talk interfering with our results. To maintain a small frequency modulation amplitude relative to $\omega_{g}$, we also reduce $q_{\mathrm{rms}}$ to $5 \times 10^{-4} e$. For this measurement we use a resolution bandwidth $B=10 \mathrm{~Hz}$.

We find a minimum charge sensitivity of $14 \mu e / \sqrt{\mathrm{Hz}}$ at $\left(n_{g}, \Phi_{\text {ext }}\right)=(0.625,0.0)$ using an input power $P_{\text {in }}=$
$-138 \mathrm{dBm} \approx 16 \mathrm{aW}$. Under these conditions our predicted theoretical and quantum-limited sensitivities are $5 \mu e / \sqrt{\mathrm{Hz}}$ and $3 \mu e / \sqrt{\mathrm{Hz}}$, respectively. The spectrum analyzer trace of this optimal measurement is shown in Fig. 2(b). At this bias point the resonant frequency is $\omega_{0} / 2 \pi=5.806 \mathrm{GHz}$, the external damping is $\kappa_{\text {ext }} / 2 \pi=1.24 \mathrm{MHz}$, and the total damping is $\kappa_{\text {tot }} / 2 \pi=1.62 \mathrm{MHz}$, such that the number of intracavity photons is $n=4 \kappa_{\text {ext }} P_{\text {in }} / \hbar \omega_{0} \kappa_{\text {tot }}^{2} \approx 1$. This singlephoton-level sensitivity is rivaled only by gate-based sensors 29, rf-SETs 21, and carbon nanotube-based rfSETs [39], all of which operate with orders of magnitude more photons. In TableII we compare the performance of the cCPT to a representative set of fast (detection bandwidth $\gtrsim 1 \mathrm{MHz})$ and ultrasensitive $\left(\delta q<10^{-3} e / \sqrt{\mathrm{Hz}}\right)$ electrometers. Clearly, the cCPT is unparalleled in its ability to operate at low powers and photon numbers. As discussed earlier, this makes it ideal for mediating an optomechanical interaction that reaches the single-photon strong coupling regime [9].

There remains significant room for improving the sensitivity of the cCPT, with two distinct approaches for doing so. The most promising approach is to reduce quasiparticle poisoning (QP) 32, which prevents us from operating at gate biases above $\left|n_{g}\right| \approx 0.65$ [30. If we were able to operate the present device at $\left(n_{g}, \Phi_{\text {ext }}\right)=$ $\left(0.9, \Phi_{0} / 2\right)$ we would expect to attain a charge sensitivity of $\delta q \approx 0.4 \mu e / \sqrt{\mathrm{Hz}}$, assuming the same factor of 3 discrepancy with theory as we observe experimentally. The present device was designed with a $9 \mathrm{~nm}$ thick CPT island [30] to suppress QP [42, but other fabrication techniques could be employed to reduce it further. These include oxygen-doping the CPT island [32] and embedding quasiparticle traps near the CPT [43]. The other approach is to mitigate the discrepancy between our measured sensitivities and the quantum limit. One such improvement would be to use a truly quantum-limited amplifier, which would improve our sensitivities by a factor of $\sqrt{S_{\text {noise }} / \hbar \omega} \approx \sqrt{2.4}$. Another such improvement would be to stabilize the resonant frequency against $1 / f$ noise using a Pound-locking loop [44. It may also be possible to improve the sensitivity of the cCPT by exploiting the nonlinearity of the cCPT [45, 46] or incorporating a parametric drive near $2 \omega_{0}$ [47].

Several important applications exist for single-photonlevel charge sensing with the cCPT. First and foremost, the cCPT can be used to dispersively sense any quantity that can be tied to electrical charge, two notable examples being the spin state of quantum-dot-based qubits and the position of a charged nanomechanical resonator. For quantum-dot-based qubits, the spin states can be encoded in charge states via spin-to-charge conversion [4, 48. For a charged nanomechanical resonator, the position of the resonator can be encoded in the charge on a capacitor [6] 8 . In both cases, the measurement backaction on the relevant degree of freedom is proportional to the number of photons in the cavity, such that single-photon-level operation is preferable [5, 35. 
Second, the cCPT can be readily integrated with nearquantum-limited amplifiers 23 25, which typically saturate well below the level of power required by rf-SETs 21, 22 and rf-QPCs 41. Finally, the cCPT has been proposed as a platform for mediating an optomechanical interaction that reaches the single-photon strong coupling regime [9]. Our demonstration of single-photonlevel electrometry with the cCPT supports the feasibility of this proposal and represents an important step toward its realization.

\section{ACKNOWLEDGMENTS}

We thank W. F. Braasch for helpful discussions and W. Oliver for providing the TWPA used in these measurements. The sample was fabricated at Dartmouth College and the Harvard Center for Nanoscale Systems. B.L.B., S.K., and A.J.R. were supported by the National Science Foundation under Grant No. DMR-1807785. J.L. was supported by the Army Research Office under Grant No. W911NF-13-1-0377. M.P.B. was supported by the National Science Foundation under Grant No. DMR1507383 .
[1] W. Lu, Z. Ji, L. Pfeiffer, K. W. West, and A. J. Rimberg, Real-time detection of electron tunnelling in a quantum dot, Nature 423, 422 (2003).

[2] O. Naaman and J. Aumentado, Time-domain measurements of quasiparticle tunneling rates in a single-cooperpair transistor, Phys. Rev. B 73, 172504 (2006).

[3] D. M. T. van Zanten, D. Sabonis, J. Suter, J. I. Väyrynen, T. Karzig, D. I. Pikulin, E. C. T. O'Farrell, D. Razmadze, K. D. Petersson, P. Krogstrup, and C. M. Marcus, Photon-assisted tunnelling of zero modes in a majorana wire, Nature Physics 16, 663 (2020).

[4] J. R. Petta, A. C. Johnson, J. M. Taylor, E. A. Laird, A. Yacoby, M. D. Lukin, C. M. Marcus, M. P. Hanson, and A. C. Gossard, Coherent manipulation of coupled electron spins in semiconductor quantum dots, Science 309, $2180(2005)$

[5] B. D'Anjou and G. Burkard, Optimal dispersive readout of a spin qubit with a microwave resonator, Physical Review B 100, 245427 (2019)

[6] R. G. Knobel and A. N. Cleland, Nanometre-scale displacement sensing using a single electron transistor, $\mathrm{Na}-$ ture 424, 291 (2003)

[7] M. D. LaHaye, O. Buu, B. Camarota, and K. C. Schwab, Approaching the quantum limit of a nanomechanical resonator, Science 304, 74 (2004).

[8] A. Naik, O. Buu, M. D. LaHaye, A. D. Armour, A. A. Clerk, M. P. Blencowe, and K. C. Schwab, Cooling a nanomechanical resonator with quantum back-action, Nature 443, 193 (2006)

[9] A. J. Rimberg, M. P. Blencowe, A. D. Armour, and P. D. Nation, A cavity-cooper pair transistor scheme for investigating quantum optomechanics in the ultra-strong coupling regime, New Journal of Physics 16, 055008 (2014)

[10] T. T. Heikkilä, F. Massel, J. Tuorila, R. Khan, and M. A. Sillanpää, Enhancing optomechanical coupling via the josephson effect, Phys. Rev. Lett. 112, 203603 (2014)

[11] J.-M. Pirkkalainen, S. Cho, F. Massel, J. Tuorila, T. Heikkilä, P. Hakonen, and M. Sillanpää, Cavity optomechanics mediated by a quantum two-level system, Nature Communications 6, 6981 (2015).

[12] X. Mi, J. V. Cady, D. M. Zajac, P. W. Deelman, and J. R. Petta, Strong coupling of a single electron in silicon to a microwave photon, Science 355, 156 (2016).

[13] X. Mi, M. Benito, S. Putz, D. M. Zajac, J. M. Taylor, G. Burkard, and J. R. Petta, A coherent spin-photon interface in silicon, Nature 555, 599 (2018).
[14] D. Zoepfl, M. Juan, C. Schneider, and G. Kirchmair, Single-photon cooling in microwave magnetomechanics, Physical Review Letters 125, 023601 (2020).

[15] P. Schmidt, M. T. Amawi, S. Pogorzalek, F. Deppe, A. Marx, R. Gross, and H. Huebl, Sideband-resolved resonator electromechanics based on a nonlinear josephson inductance probed on the single-photon level, Communications Physics 3, 233 (2020).

[16] M. Kounalakis, Y. M. Blanter, and G. A. Steele, Fluxmediated optomechanics with a transmon qubit in the single-photon ultrastrong-coupling regime, Physical Review Research 2, 023335 (2020).

[17] T. Bera, S. Majumder, S. K. Sahu, and V. Singh, Large flux-mediated coupling in hybrid electromechanical system with a transmon qubit, Communications Physics 4, $12(2021)$

[18] A. Nunnenkamp, K. Børkje, and S. M. Girvin, Singlephoton optomechanics, Physical Review Letters 107, 063602 (2011)

[19] P. Rabl, Photon blockade effect in optomechanical systems, Physical Review Letters 107, 063601 (2011).

[20] M. Aspelmeyer, T. J. Kippenberg, and F. Marquardt, Cavity optomechanics, Reviews of Modern Physics 86, 1391 (2014).

[21] H. Brenning, S. Kafanov, T. Duty, S. Kubatkin, and P. Delsing, An ultrasensitive radio-frequency singleelectron transistor working up to $4.2 \mathrm{k}$, Journal of Applied Physics 100, 114321 (2006)

[22] R. J. Schoelkopf, P. Wahlgren, A. A. Kozhevnikov, P. Delsing, and D. E. Prober, The radio-frequency singleelectron transistor (rf-set): A fast and ultrasensitive electrometer, Science 280, 1238 (1998)

[23] M. A. Castellanos-Beltran and K. W. Lehnert, Widely tunable parametric amplifier based on a superconducting quantum interference device array resonator, Applied Physics Letters 91, 083509 (2007)

[24] C. Macklin, K. O'Brien, D. Hover, M. E. Schwartz, V. Bolkhovsky, X. Zhang, W. D. Oliver, and I. Siddiqi, A near-quantum-limited josephson traveling-wave parametric amplifier, Science 350, 307 (2015).

[25] V. Sivak, N. Frattini, V. Joshi, A. Lingenfelter, S. Shankar, and M. Devoret, Kerr-free three-wave mixing in superconducting quantum circuits, Physical Review Applied 11, 054060 (2019).

[26] M. A. Sillanpää, L. Roschier, and P. J. Hakonen, Inductive single-electron transistor, Physical Review Letters 
93, 066805 (2004).

[27] M. A. Sillanpää, L. Roschier, and P. J. Hakonen, Charge sensitivity of the inductive single-electron transistor, Applied Physics Letters 87, 092502 (2005).

[28] M. F. Gonzalez-Zalba, S. Barraud, A. J. Ferguson, and A. C. Betz, Probing the limits of gate-based charge sensing, Nature Communications 6, 6084 (2015).

[29] S. Schaal, I. Ahmed, J. Haigh, L. Hutin, B. Bertrand, S. Barraud, M. Vinet, C.-M. Lee, N. Stelmashenko, J. Robinson, J. Qiu, S. Hacohen-Gourgy, I. Siddiqi, M. Gonzalez-Zalba, and J. Morton, Fast gate-based readout of silicon quantum dots using josephson parametric amplification, Physical Review Letters 124, 067701 (2020)

[30] B. L. Brock, J. Li, S. Kanhirathingal, B. Thyagarajan, W. F. Braasch, M. P. Blencowe, and A. J. Rimberg, Nonlinear charge- and flux-tunable cavity derived from an embedded cooper-pair transistor, Phys. Rev. Applied 15, 044009 (2021)

[31] S. Kanhirathingal, B. L. Brock, A. J. Rimberg, and M. P. Blencowe, Charge sensitivity of a cavity-embedded cooper pair transistor limited by single-photon shot noise, Journal of Applied Physics 130, 114401 (2021)

[32] J. Aumentado, M. W. Keller, J. M. Martinis, and M. H. Devoret, Nonequilibrium quasiparticles and $2 e$ periodicity in single-cooper-pair transistors, Physical Review Letters 92, 066802 (2004)

[33] D. Pozar, Microwave Engineering (Wiley, 2004).

[34] A. Aassime, D. Gunnarsson, K. Bladh, P. Delsing, and R. Schoelkopf, Radio-frequency single-electron transistor: Toward the shot-noise limit, Applied Physics Letters 79, 4031 (2001).

[35] A. A. Clerk, M. H. Devoret, S. M. Girvin, F. Marquardt, and R. J. Schoelkopf, Introduction to quantum noise, measurement, and amplification, Reviews of Modern Physics 82, 1155 (2010).

[36] E. Paladino, Y. M. Galperin, G. Falci, and B. L. Altshuler, 1/f noise: Implications for solid-state quantum information, Reviews of Modern Physics 86, 361 (2014)

[37] C. M. Caves, Quantum limits on noise in linear amplifiers, Phys. Rev. D 26, 1817 (1982).

[38] B. L. Brock, M. P. Blencowe, and A. J. Rimberg, Frequency fluctuations in tunable and nonlinear microwave cavities, Phys. Rev. Applied 14, 054026 (2020).

[39] S. E. S. Andresen, F. Wu, R. Danneau, D. Gunnarsson, and P. J. Hakonen, Highly sensitive and broadband carbon nanotube radio-frequency single-electron transistor, Journal of Applied Physics 104, 033715 (2008).

[40] M. T. Bell, L. B. Ioffe, and M. E. Gershenson, Microwave spectroscopy of a cooper-pair transistor coupled to a lumped-element resonator, Physical Review B 86, 144512 (2012)

[41] M. C. Cassidy, A. S. Dzurak, R. G. Clark, K. D. Petersson, I. Farrer, D. A. Ritchie, and C. G. Smith, Single shot charge detection using a radio-frequency quantum point contact, Applied Physics Letters 91, 222104 (2007).

[42] T. Yamamoto, Y. Nakamura, Y. A. Pashkin, O. Astafiev, and J. S. Tsai, Parity effect in superconducting aluminum single electron transistors with spatial gap profile controlled by film thickness, Applied Physics Letters 88, 212509 (2006)

[43] S. Rajauria, L. M. A. Pascal, P. Gandit, F. W. J. Hekking, B. Pannetier, and H. Courtois, Efficiency of quasiparticle evacuation in superconducting devices, Physical Review B 85, 020505 (2012).

[44] T. Lindström, J. Burnett, M. Oxborrow, and A. Y. Tzalenchuk, Pound-locking for characterization of superconducting microresonators, Review of Scientific Instruments 82, 104706 (2011)

[45] C. Laflamme and A. A. Clerk, Quantum-limited amplification with a nonlinear cavity detector, Physical Review A 83, 033803 (2011)

[46] L. Tosi, D. Vion, and H. le Sueur, Design of a cooper-pair box electrometer for application to solid-state and astroparticle physics, Physical Review Applied 11, 054072 (2019).

[47] P. Krantz, A. Bengtsson, M. Simoen, S. Gustavsson, V. Shumeiko, W. D. Oliver, C. M. Wilson, P. Delsing, and J. Bylander, Single-shot read-out of a superconducting qubit using a josephson parametric oscillator, Nature Communications 7, 11417 (2016)

[48] R. Hanson, L. P. Kouwenhoven, J. R. Petta, S. Tarucha, and L. M. K. Vandersypen, Spins in few-electron quantum dots, Reviews of Modern Physics 79, 1217 (2007) 